PROCEEDINGS OF THE AMERICAN MATHEMATICAL SOCIETY

Volume 124, Number 6, June 1996

\title{
INVARIANT SUBSPACES OF THE HARMONIC DIRICHLET SPACE WITH LARGE CO-DIMENSION
}

\author{
WILLIAM T. ROSS
}

(Communicated by Palle E. T. Jorgensen)

\begin{abstract}
In this paper, we comment on the complexity of the invariant subspaces (under the bilateral Dirichlet shift $f \rightarrow \zeta f$ ) of the harmonic Dirichlet space $D$. Using the sampling theory of Seip and some work on invariant subspaces of Bergman spaces, we will give examples of invariant subspaces $\mathcal{F} \subset D$ with $\operatorname{dim}(\mathcal{F} / \zeta \mathcal{F})=n, n \in \mathbb{N} \cup\{\infty\}$. We will also generalize this to the Dirichlet classes $D_{\alpha}, 0<\alpha<\infty$, as well as the Besov classes $B_{p}^{\alpha}, 1<p<\infty$, $0<\alpha<1$.
\end{abstract}

\section{INTRODUCTION}

For a space $X$ of functions on the unit circle $\mathbb{T}=\{z \in \mathbb{C}:|z|=1\}$ for which the shift operator

$$
T: X \rightarrow X,(T f)(\zeta)=\zeta f(\zeta)
$$

is continuous and invertible (e.g. $L^{p}(\mathbb{T},|d \zeta|), C^{n}(\mathbb{T}), C^{\infty}(\mathbb{T}), W_{n}^{p}(\mathbb{T})$ the Sobolev classes, $B_{p}^{\alpha}(\mathbb{T})$ the Besov classes, $D_{\alpha}$ the Dirichlet classes, $B L_{p, \theta}^{l}(\mathbb{T})$ the TriebelLizorkin classes), the problem of characterizing the subspaces (closed linear manifolds) $\mathcal{F} \subset X$ for which $T \mathcal{F} \subset \mathcal{F}$, the so called invariant subspaces, is a very difficult and open problem. There are two types of invariant subspaces to consider: simply invariant (or 1-invariant) $T \mathcal{F} \neq \mathcal{F}$, and 2 -invariant $T \mathcal{F}=\mathcal{F}$. The 2 -invariant subspaces are often described by their zero sets in $\mathbb{T}[1]$, [10], [11], [12], while the 1-invariant subspaces are known to be much more complicated and for most of the classes mentioned above, a complete characterization seems a long way off [3], [6], $[8],[9]$.

In this paper, we focus our attention on the harmonic Dirichlet space $D$ of functions $f \in L^{2}(\mathbb{T},|d \zeta|)$ with finite norm

$$
\|f\|^{2}=\sum_{n=-\infty}^{\infty}(1+|n|)|\hat{f}(n)|^{2},
$$

where $\{\hat{f}(n)\}$ are the Fourier coefficients of $f$. The 2-invariant subspaces of $D$ can be characterized by their zero sets on $\mathbb{T}[12]$, while the simply invariant subspaces of $D$ are much more complicated. In this paper, we remark on the complexity of the invariant subspaces $\mathcal{F}$ with $D_{A} \subset \mathcal{F} \subset D$, where $D_{A}$ is the analytic Dirichlet

Received by the editors October 31, 1994 and, in revised form, December 9, 1994.

1991 Mathematics Subject Classification. Primary 30H05; Secondary 30C15.

Key words and phrases. Dirichlet spaces, invariant subspaces, co-dimension, Bergman spaces.

This research was supported in part by a grant from the National Science Foundation. 
space $\{f \in D: \hat{f}(n)=0 \forall n<0\}$, by examining their index. Here for an invariant subspace $\mathcal{F} \subset D$, we define the index (or sometimes called the co-dimension) to be

$$
\text { ind } \mathcal{F}=\operatorname{dim}(\mathcal{F} / \zeta \mathcal{F}) \text {. }
$$

For the analytic Dirichlet space $D_{A}$, every non-zero invariant subspace has index 1 [13]. In stark contrast to this, it was observed in [1] that there are examples of invariant subspaces $D_{A} \subset \mathcal{F} \subset D$ with ind $\mathcal{F}=n$ for any $n \in \mathbb{N} \cup\{\infty\}$. We will give a new and more direct proof of this fact as well as specific examples of these types of subspaces. To do this, we will employ the natural one-to-one correspondence between the invariant subspaces $D_{A} \subset \mathcal{F} \subset D$ and the invariant subspaces (under $f \rightarrow z f$ ) of the Bergman space $L_{a}^{2}(\mathbb{D})$. We will then use some recent results of Seip, Hedenmalm, and Richter [4], [5] ,[14] which yield specific examples of invariant subspaces of the Bergman space with large index. We then transfer this information back to the Dirichlet space to obtain the following theorem.

Theorem 1.1. Given $n \in \mathbb{N} \cup\{\infty\}$, there are sequences $A_{j}, 0 \leq j<n$, of points in $\mathbb{D}$ such that

$$
\mathcal{F}=\bigcap_{0 \leq j<n} \operatorname{span}\left\{\overline{B_{a}} H^{2} \cap D: a \in A_{j}\right\}
$$

is a simply invariant subspace of $D$ with ind $\mathcal{F}=n$.

Note that $H^{2}=\left\{f \in L^{2}: \hat{f}(n)=0 \quad \forall n<0\right\}$ is the usual Hardy space on the circle and

$$
B_{a}(\zeta)=\frac{\bar{a}}{|a|} \frac{a-\zeta}{1-\bar{a} \zeta}, \quad \zeta \in \mathbb{T} .
$$

We also use span $X$ to denote the closed linear span of $X$, and $\bar{z}$ to denote the complex conjugate of a complex number $z$.

We will remark at the end that a similar result also holds for the Dirichlet spaces $D_{\alpha}$ and the Besov classes $B_{p}^{\alpha}(\mathbb{T})$.

\section{BERgman SPACES}

The sequences $A_{j}$ in Theorem 1.1 will be zero sequences of the Bergman space, and thus we begin our discussion with some basic Bergman space facts. The Bergman space $L_{a}^{2}(\mathbb{D})$ is the space of analytic functions $f$ on $\mathbb{D}$ such that

$$
\int_{\mathbb{D}}|f(z)|^{2} d A(z)=\sum_{n=0}^{\infty} \frac{\left|a_{n}\right|^{2}}{n+1}<\infty .
$$

Here $d A$ is area measure on $\mathbb{D}$ normalized so it has mass 1 , and $\left\{a_{n}\right\}$ are the power series coefficients of $f$. It is well known that $L_{a}^{2}(\mathbb{D})$ is a closed subspace of $L^{2}(\mathbb{D}, d A)$ and the operator $f \rightarrow z f$ is continuous. The subspaces $\mathcal{M} \subset L_{a}^{2}(\mathbb{D})$ with $z \mathcal{M} \subset \mathcal{M}$ (also called invariant subspaces) are tremendously complicated and poorly understood. As before, we define the index (or co-dimension) of an invariant subspace $\mathcal{M} \subset L_{a}^{2}(\mathbb{D})$ to be ind $\mathcal{M}=\operatorname{dim}(\mathcal{M} / z \mathcal{M})$. It is known [2] that given $n \in \mathbb{N} \cup\{\infty\}$ there exists an invariant subspace $\mathcal{M} \subset L_{a}^{2}(\mathbb{D})$ with ind $\mathcal{M}=n$. Moreover, using sampling theory, Hedenmalm, Richter, and Seip [4], [5] have been able to generate specific examples of these types of invariant subspaces by using zero-based invariant subspaces.

Given a sequence $A \subset \mathbb{D}$, we let

$$
\mathcal{I}(A)=\left\{f \in L_{a}^{2}(\mathbb{D}):\left.f\right|_{A}=0\right\} .
$$


In the case where $\mathcal{I}(A) \neq 0$ one can show that $\mathcal{I}(A)$ is a closed invariant subspace of $L_{a}^{2}(\mathbb{D})$ with ind $\mathcal{I}(A)=1$. We now state the Seip, Hedenmalm, Richter result [4], [5].

Theorem 2.1 (Hedenmalm, Richter, Seip). Given $n \in \mathbb{N} \cup\{\infty\}$ there are sequences $A_{j}, 0 \leq j<n$, of points in $\mathbb{D}$ such that

$$
\mathcal{M}=\operatorname{span}\left\{\mathcal{I}\left(A_{j}\right): 0 \leq j<n\right\}
$$

is an invariant subspace of $L_{a}^{2}(\mathbb{D})$ with ind $\mathcal{M}=n$.

\section{ThE CORRESPONDENCE}

As mentioned in the introduction, there will be a natural correspondence between the invariant subspaces $D_{A} \subset \mathcal{F} \subset D$ and the invariant subspaces of the Bergman space. This type of correspondence was observed by Makarov [9] in the $C^{\infty}(\mathbb{T})$ case where he observed a natural correspondence between the invariant subspaces $C_{A}^{\infty}(\mathbb{T}) \subset \mathcal{F} \subset C^{\infty}(\mathbb{T})$ and the invariant subspaces (under $f \rightarrow z f$ ) of $A^{-\infty}$, the analytic distributions. Here $C_{A}^{\infty}(\mathbb{T})=\left\{f \in C^{\infty}: \hat{f}(n)=0 \forall n<0\right\}$, and $A^{-\infty}$ are the distributions $g$ with $\hat{g}(n)=0$ for all $n<0$. We make a similar observation for the Dirichlet space and also prove an interesting index formula.

The dual space of $D$, which we will denote by $D^{\prime}$, is the space of distributions $g$ with

$$
\sum_{n=-\infty}^{\infty} T \infty \frac{|\hat{g}(n)|^{2}}{|n|+1}<\infty
$$

The pairing between $D$ and $D^{\prime}$ is given by

$$
\langle f, g\rangle=\sum_{n=-\infty}^{\infty} \hat{f}(n) \overline{\hat{g}(n)}, \quad f \in D, g \in D^{\prime} .
$$

Let $D_{A}^{\prime}$ be the analytic distributions of $D^{\prime}$

$$
D_{A}^{\prime}=\left\{g \in D^{\prime}: \hat{g}(n)=\left\langle\zeta^{n}, g\right\rangle=0 \quad \forall n<0\right\} .
$$

Making $\{\hat{g}(n)\}$ the power series coefficients of an analytic function $g(z)$ on the disk, we see that $D_{A}^{\prime}$ can be naturally identified with the Bergman space $L_{a}^{2}(\mathbb{D})$ and moreover

$$
\langle f, g\rangle=\lim _{r \rightarrow 1^{-}} \int_{\mathbb{T}} f(r \zeta) \overline{g(r \zeta)} \frac{|d \zeta|}{2 \pi}, \quad f \in D, g \in D_{A}^{\prime} .
$$

Theorem 3.1. (1) If $D_{A} \subset \mathcal{F} \subset D$ is invariant, then $\mathcal{M}=(\overline{\zeta \mathcal{F}})^{\perp}$ is an invariant subspace of $L_{a}^{2}(\mathbb{D})$.

(2) If $\mathcal{M} \subset L_{a}^{2}(\mathbb{D})$ is invariant, then $\mathcal{F}=\bar{\zeta}(\overline{\mathcal{M}})^{\perp}$ is invariant and $D_{A} \subset \mathcal{F} \subset D$.

(3) ind $\mathcal{M}=$ ind $\mathcal{F}$.

Proof. (1) Letting $g \in \mathcal{M}$, we see that $\langle\overline{\zeta f}, g\rangle=0$ for all $f \in \mathcal{F}$. But since $\zeta^{n} \in \mathcal{F}$ for all $n \geq 0$, then $\left\langle\bar{\zeta}^{n+1}, g\right\rangle=\hat{g}(-n-1)=0$ for all $n \geq 0$, i.e. $g \in D_{A}^{\prime}=L_{a}^{2}(\mathbb{D})$. Also, by (3.1),

$$
\langle\overline{\zeta f}, z g\rangle=\langle\bar{\zeta}(\overline{\zeta f}), g\rangle=0 \quad \forall f \in \mathcal{F}
$$

since $\mathcal{F}$ is invariant. Thus $z g \in \mathcal{M}$, which makes $\mathcal{M}$ an invariant subspace of $L_{a}^{2}(\mathbb{D})$.

(2) If $f \in \mathcal{F}$, then $\zeta f \in(\overline{\mathcal{M}})^{\perp}$ and so

$$
\langle\zeta(\zeta f), \bar{g}\rangle=\langle\zeta f, \overline{z g}\rangle=0 \quad \forall g \in \mathcal{M}
$$


since $\mathcal{M}$ is invariant. Thus $\zeta f \in \bar{\zeta}(\overline{\mathcal{M}})^{\perp}=\mathcal{F}$. Also, $\mathcal{M} \subset L_{a}^{2}(\mathbb{D})=D_{A}^{\prime}$ and so

$$
0=\left\langle\zeta^{n+1}, \bar{g}\right\rangle=\left\langle\zeta \zeta^{n}, \bar{g}\right\rangle \quad \forall n \geq 0, g \in \mathcal{M},
$$

which implies $\zeta^{n} \in \bar{\zeta}(\overline{\mathcal{M}})^{\perp}=\mathcal{F}$. Thus $D_{A} \subset \mathcal{F} \subset D$ and $\mathcal{F}$ is invariant.

(3) To prove the index formula, first we notice from the Hahn-Banach theorem and basic linear algebra that

$$
\begin{aligned}
\operatorname{dim}(\mathcal{F} / \zeta \mathcal{F}) & =\operatorname{dim}\left((\zeta \mathcal{F})^{\perp} / \mathcal{F}^{\perp}\right) \\
& =\operatorname{dim}\left(\overline{\mathcal{M}} /\left(\bar{\zeta}\left(\overline{\mathcal{M}}^{\perp}\right)\right)^{\perp}\right) \\
& =\operatorname{dim}\left(\mathcal{M} /\left(\zeta\left(\mathcal{M}^{\perp}\right)\right)^{\perp}\right)
\end{aligned}
$$

The proof will be finished once we show that

$$
\left(\zeta\left(\mathcal{M}^{\perp}\right)\right)^{\perp}=z \mathcal{M} .
$$

To this end, we let $g \in \mathcal{M}$; then $z g \in \mathcal{M}$ and for all $f \in \mathcal{M}^{\perp}$,

$$
\langle\zeta f, z g\rangle=\langle\bar{\zeta} \zeta f, g\rangle=\langle f, g\rangle=0 .
$$

Thus $z g \in\left(\zeta\left(\mathcal{M}^{\perp}\right)\right)^{\perp}$.

For the other direction we observe that

$$
D_{A} \subset \mathcal{F}=\bar{\zeta}(\overline{\mathcal{M}})^{\perp}
$$

and so $\overline{\zeta D_{A}} \subset \mathcal{M}^{\perp}$. Thus if $g \in\left(\zeta\left(\mathcal{M}^{\perp}\right)\right)^{\perp}$, then

$$
0=\langle g, \zeta \bar{\zeta}\rangle=\langle g, 1\rangle=\hat{g}(0) .
$$

Next we observe (using the invariance of $\mathcal{M}$ ) that if $h \in \mathcal{M}^{\perp}$,

$$
0=\langle h, k\rangle=\langle h, z k\rangle=\langle\bar{\zeta} h, k\rangle \quad \forall k \in \mathcal{M} .
$$

Thus $\bar{\zeta} h \in \mathcal{M}^{\perp}$. Hence $0=\langle g, \zeta \bar{\zeta} h\rangle=\langle g, h\rangle$ for all $h \in \mathcal{M}^{\perp}$. Thus $g \in\left(\mathcal{M}^{\perp}\right)^{\perp}=$ $\mathcal{M}$. Now using the fact that $g \in L_{a}^{2}(\mathbb{D})$ and $g(0)=0$, we get that $\frac{1}{z} g \in L_{a}^{2}(\mathbb{D})$ and so, by (3.1),

$$
0=\langle g, \zeta h\rangle=\left\langle z \frac{1}{z} g, \zeta h\right\rangle=\left\langle\frac{1}{z} g, h\right\rangle \quad \forall h \in \mathcal{M}^{\perp} .
$$

Thus $\frac{1}{z} g \in \mathcal{M}$ and hence $g \in z \mathcal{M}$. So (3.2) has been established and the proof is complete.

Recall from (1.1) that for $w \in \mathbb{D}, B_{w}(\zeta)$ is the single Blaschke factor with zero at $w$. Using the fact that $\mathcal{I}(\{w\})=\operatorname{span}\left\{z^{n} B_{w}(z): n \geq 0\right\}$, and the F. and M. Riesz theorem, one can prove the following.

Lemma 3.2. If $\mathcal{M}=\mathcal{I}(\{w\})$, then $\mathcal{F}=\bar{\zeta}(\overline{\mathcal{M}})^{\perp}=\overline{B_{w}} H^{2} \cap D$.

We are now ready to prove our main theorem.

Proof of Theorem 1.1. By Theorem 2.1, the invariant subspace

$$
\mathcal{M}=\operatorname{span}\left\{\mathcal{I}\left(A_{j}\right): 0 \leq j<n\right\}
$$


has index $n$. Thus by Theorem $3.1, \mathcal{F}=\bar{\zeta}(\overline{\mathcal{M}})^{\perp}$ also has index $n$. We now identify $\mathcal{F}$ :

$$
\begin{aligned}
\mathcal{F} & =\bar{\zeta}(\overline{\mathcal{M}})^{\perp} \\
& =\bar{\zeta}\left(\operatorname{span}\left\{\overline{\mathcal{I}\left(A_{j}\right)}: 0 \leq j<n\right\}\right)^{\perp} \\
& \left.=\bar{\zeta}\left(\bigcap_{0 \leq j<n} \overline{\mathcal{I}\left(A_{j}\right)}\right)^{\perp}\right) \\
& =\bigcap_{0 \leq j<n} \bar{\zeta}\left(\bigcap_{a \in A_{j}} \overline{\mathcal{I}(\{a\})}\right)^{\perp} \quad \text { since mult. by } \bar{\zeta} \text { is invertible } \\
& =\bigcap_{0 \leq j<n} \bar{\zeta} \operatorname{span}\left\{\overline{\mathcal{I}(\{a\})^{\perp}}: a \in A_{j}\right\} \\
& =\bigcap_{0 \leq j<n} \operatorname{span}\left\{\bar{\zeta}\left(\overline{\mathcal{I}(\{a\})^{\perp}}\right): a \in A_{j}\right\} \quad \text { since mult. by } \zeta \text { is cont. and invertible } \\
& =\bigcap_{0 \leq j<n} \operatorname{span}\left\{\overline{B_{a}} H^{2} \cap D: a \in A_{j}\right\} \quad \text { by Lemma } 3.2 .
\end{aligned}
$$

\section{Generalizations}

In this last section, we remark that the analog of Theorem 1.1 is also true for the Dirichlet classes $D_{\alpha}, 0<\alpha<\infty$, of $f \in L^{2}(\mathbb{T},|d \zeta|)$ with

$$
\sum_{n=-\infty}^{\infty}(1+|n|)^{\alpha}|\hat{f}(n)|^{2}<\infty
$$

and the Besov classes $B_{p}^{\alpha}, 1<p<\infty, 0<\alpha<1$, of $f$ with

$$
\int_{0}^{\pi} \int_{0}^{2 \pi} \frac{\left|f\left(e^{i(\theta+h)}\right)-f\left(e^{i \theta}\right)\right|^{p}}{h^{1+\alpha p}} d \theta d h<\infty
$$

Here the appropriate Bergman spaces to consider are the weighted Bergman spaces $A_{p}^{\alpha}, 0<\alpha<\infty, 1<p<\infty$, of analytic functions on $\mathbb{D}$ with

$$
\int_{\mathbb{D}}|f(z)|^{p}(1-|z|)^{\alpha p-1} d A(z)<\infty .
$$

The analog of Theorem 2.1 is true for $A_{p}^{\alpha}$ where, of course, $\mathcal{I}(A)=\left\{f \in A_{p}^{\alpha}:\left.f\right|_{A}=\right.$ $0\}$ [4], [5]. For $D_{\alpha}$, the analytic distributions (in the dual) can be identified with the weighted Bergman space $A_{2}^{\alpha / 2}$ via (3.1) [7]. For $B_{p}^{\alpha}$, the analytic distributions (in the dual) can be identified with $A_{q}^{\alpha}$, where $q=p(p-1)^{-1}$ via (3.1). For both these spaces, the analog of Theorem 3.1 remains true. Thus we have the following.

Theorem 4.1. Let $X=D_{\alpha}, 0<\alpha<\infty$, or $B_{p}^{\alpha}, 1<p<\infty, 0<\alpha<1$, and $n \in \mathbb{N} \cup\{\infty\}$. Then there are sequences $A_{j}, 0 \leq j<n$, of points in $\mathbb{D}$ so that

$$
\mathcal{F}=\bigcap_{0 \leq j<n} \operatorname{span}_{X}\left\{\overline{B_{a}} H^{2} \cap X: a \in A_{j}\right\}
$$

is a simply invariant subspace of $X$ with ind $\mathcal{F}=n$. 


\section{REFERENCES}

[1] A. Aleman, S. Richter, and W.T. Ross, 'Bergman spaces on disconnected domains, Canad. J. Math. (to appear).

[2] H. Bercovici, C. Foiaş, and C. Pearcy, Dual algebras with applications to invariant subspaces and dilation theory, CBMS Regional Conf. Ser. in Math., no. 56, Amer. Math. Soc., Providence, RI., 1985. MR 87g:47091

[3] M. Hasumi and T.P. Srinivasan, 'Invariant subspaces of continuous functions', Canad. J. Math. 22 (1965), 643 - 651. MR 31:3871

[4] H. Hedenmalm, 'An invariant subspace of the Bergman space having the co-dimension two property', J. Reine Angew. Math. 443 (1993), 1 - 9. MR 94k:30092

[5] H. Hedenmalm, S. Richter, K. Seip, 'Zero sequences and invariant subspaces in the Bergman space', preprint.

[6] H. Helson, Lectures on invariant subspaces, New York and London, 1964. MR 30:1409

[7] S. Khrushchev and V. Peller, 'Hankel operators, best approximation, and stationary Gaussian processes', Russian Math. Surveys, 37 (1982), 61 - 144. MR 84e:47036

[8] N.G. Makarov, 'Sets of 1-invariant and and 1-invariant subspaces (smooth functions)', Soviet Math. Dokl. 25 (1982), 191 - 194. MR 83h:46053

[9] N.G. Makarov, 'Invariant subspaces of the space $C^{\infty}(\mathbb{T})$, Math. USSR Sbornik, 47 (1984), 1 - 26. MR 84d:30088

[10] B. Malgrange, Ideals of differentiable functions, Tata Inst. Fund. Res., Bombay and Oxford Univ. Press, London, 1967. MR 35:3446

[11] Y.U. Netrusov 'Spectral synthesis in spaces of smooth functions', Russian Acad. Dokl. Math., 46 (1993), 135 - 138. MR 94c:46068

[12] S. Richter, W. T. Ross, and C. Sundberg, 'Hyperinvariant subspaces of the harmonic Dirichlet space', J. Reine Angew. Math. 448 (1994), 1 - 26. MR 95e:47045

[13] S. Richter and A. Shields, 'Bounded analytic functions in the Dirichlet space', Math. Z. 198 (1988), 151 - 159. MR 89c:46039

[14] K. Seip, 'Beurling type density theorems in the unit disk', Invent. Math. 113 (1993), 26 - 39. MR 94g:30033

Department of Mathematics, University of Richmond, Richmond, Virginia 23173

E-mail address: rossb@mathcs.urich.edu 